Psychiatry of Mental Handicap Lynebank Hospital Dunfermline

\section{Fluoxetine and suicidal behaviour}

SIR: Power \& Cowen (Journal, December 1992, 161, 735-741) have performed a comprehensive overview of available data. However, it is of concern that they believe many clinicians are still disquietened despite the balance of evidence suggesting there is no association between fluoxetine and suicidal behaviour. The quote from an American psychiatrist merely highlights the media involvement and the fact that medical care in the USA may be compromised by threats of litigation.

Both the Committee on Safety of Medicines (CSM) and the Food and Drug Administration (FDA) have issued statements on fluoxetine. In September 1991 the FDA reviewed all the data and concluded that there was "no credible evidence" to link fluoxetine to suicidal or aggressive behaviour. A recent CSM Current Problems (1992) and a Manufacturing Chemists Association (MCA)/CSM paper (Price et al, 1992) also lend extra weight to this, stating "there is little to support the suggestion that fluoxetine induces suicidal or aggressive behaviour".

Drs Power and Cowen rightly concentrate on the scientific aspects of the controversy. They remark on the fact that development of suicidal ideation in patients taking antidepressants is not new. In the Damluji and Ferguson (1988) case studies two of four patients on desipramine who were subsequently switched to fluoxetine made a complete recovery with no recurrence of suicidal ideation.

The hypothesis of a rare idiosyncratic reaction, perhaps in the context of induced akathisia, is proposed. The data available to support this hypothesis are far from established. A recent paper by Baldessarini et al (1992) failed to show a dopamine-inhibiting effect of fluoxetine either with acute or repeated doses. The suggestion is also made that, of the selective serotonin (5-hydroxytryptamine, 5-HT) reuptake inhibitors (SSRIs), fluoxetine is most activating. Although data varies (and most work has been done at $\mathbf{4 0} \mathrm{mg}$ ) it is, in fact, not the case. Kerr \& Hindmarch (1991), in their review of the cognitive and behavioural effects of the 5-HT reuptake inhibitors, conclude that these drugs are essentially neutral.

We will continue to be vigilant in our collection of safety data for fluoxetine. As the SSRIs in general become more commonly used, it may be that specific effects become more clearly delineated and clinicians will learn to manage these as they have those of the older tricyclic antidepressants which are arguably more toxic to patients.

Baldessarin, R. J., Marsh, E. R. \& Kula, N. S. (1992) Interactions of fluoxetine with metabolism of dopamine and serotonin in rat brain regions. Brain Research, 579, 152-156.

COMmrttee on SAFETy of Medicines (1992) Safety of fluoxetine (Prozac): Comparison with fluvoxamine (Faverin). Current Problems (June).

Damlun, N. F. \& Ferguson, J. M. (1988) Paradoxical worsening of depressive symptomatology caused by antidepressants. Journal of Clinical Psychopharmacology, 8, 347-349.

KerR, J. S., HindmarCH, I. \& SherwOOd, N. (1991) The comparative psychopharmacology of 5-HT reuptake inhibitors. Human Psychopharmacology, 6, 313-317.

Pruce, J., et al (1992) Safety of fluoxetine: comparison with fluvoxamine. Pharmacoepidemiology and Drug Safety, 1, 111-117.

Clinical Research Physician

DEBBiE HARRISON

Lilly Industries Limited

Dextra Court, Chapel Hill

Basingstoke

Hampshire RG21 2SY

\section{Periodic psychosis associated with the menstrual} cycle and childbirth

SIR: I agree with Crammer (Journal, December 1992, $161,859)$ that the relationship between mental states and monthly cycles is complex, and add that a better understanding of this relationship may help untangle the biological basis of certain mental disorders. However, I am sorry that the relationship between the mental state and the menstrual cycle was not more convincing in my case report (Journal, September 1992, 161, 402-404). The graph presents an accurate record of the timing of the patient's mental state and menses, as far as it is possible to demonstrate such things pictorially. As stated in the text, three of six psychotic episodes began in the week prior to menses, and two in the fortnight before.

Sometime after writing the report, my patient moved and was unfortunately lost to follow-up. It later transpired that she did not take any medication after losing contact. During this period she had a stable relationship and became pregnant, sustaining the longest period of good health that she had enjoyed for a number of years. Both she and her relatives were so struck by the disappearance of her regular relapses that they believed she was now free from her illness. After a full-term and uncomplicated delivery she returned home. However, three days later she disclosed to a midwife ideas about killing the baby. On the fourth post-partum day she was admitted to a local psychiatric unit, perplexed, anxious, and experiencing accusatory auditory 